\title{
Crisis y apocalipsis. Notas para un análisis de la reflexión scholemiana sobre el mesianismo
}

\section{Crisis and Apocalypse. Notes towards an Analysis of Gershom Scholem's Reflection on Messianism}

\author{
Enrico LuCCA \\ Franz Rosenzweig Minerva Research Center \\ (The Hebrew University of Jerusalem) \\ en.lucca@gmail.com
}

Recibido: $18 / 01 / 2015$

Aceptado: 24/06/2015

\section{Resumen}

En este artículo se propone explorar la lectura del mesianismo judío ofrecida por el gran historiador de la Qabbalah Gershom Scholem (1897-1982). En primer lugar se muestra cómo el mesianismo ha marcado profundamente la investigación de Scholem desde el principio de su actividad académica. Posteriormente se busca esclarecer las líneas principales en la descripción scholemiana del fenómeno mesiánico: su oposición a la concepción positivista y neokantiana de la historia, su predilección por una interpretación apocalíptica del mesianismo judío y su fascinación por movimientos radicales como el sabatianismo y el frankismo. Finalmente se ofrece un análisis de las críticas de Jacob Taubes (1923-1987) a la visión scholemiana del mesianismo.

Palabras clave: Gershom Scholem, mesianismo, apocalipsis, crisis, judaísmo alemán, Jacob Taubes

\begin{abstract}
This paper aims to explore the reading of Jewish messianism provided by the great historian of Jewish mysticism Gershom Scholem (1897-1982). Starting by showing how messianism has been at the center of Scholem's attention since the beginning of his scholarly activity, the main motives at the core of Scholem's description of the messianic phenomenon are investigated. The article points out Scholem's opposition to a positivist and neo-kantian conception of history, his predilection for the apocalyptical interpretation of Jewish messianism, and his fascination for radical
\end{abstract}


movements such as Sabbatianism and Frankism. In the final part an explanation of the main critiques moved against Scholem's view of messianism by Jacob Taubes (1923-1987) is also offered.

Keywords: Gershom Scholem, messianism, apocalypse, crisis, German-Jewry, Jacob Taubes

La investigación de Gershom Scholem en el ámbito del misticismo judío representa sin duda uno de los momentos más importantes de la historia de las religiones del siglo xx. El hecho de que Scholem, según una afirmación atribuida a Martin Buber, haya sido el único pensador capaz de instaurar no sólo una escuela, sino una entera disciplina de estudios, es muy significativo y representa de algún modo el reconocimiento de la importancia absoluta de Scholem - que dejó Berlín para emigrar a Jerusalén con apenas veintiséis años - y de su papel fundamental en el panorama intelectual del judaísmo del siglo pasado.

La publicación de textos inéditos después de su muerte y, en particular, de los dos volúmenes de diarios que retratan el periodo entre 1913 y $1923^{1}$, ha puesto en evidencia que ya desde su juventud, si bien de forma aún no del todo madura, Scholem tenía bien clara cuál sería la dirección futura de sus investigaciones. Scholem empezó estudiando matemáticas en Jena, donde tuvo también la oportunidad de frecuentar los cursos de Frege. Más tarde, decidió dedicarse enteramente al estudio de la Qabbalah, empujado por una urgencia "metafísica", como él mismo reconoció algunos años después. Al mismo tiempo, sin embargo, el entusiasmo juvenil que le llevó a ocuparse de los distintos aspectos de la tradición judía fue siempre acompañado por un uso muy riguroso del método histórico y filológico. Por otro lado, es cierto igualmente que la investigación de Scholem sobre la Qabbalah procedía de un fuerte espíritu polémico, que por supuesto no conllevaba un rechazo total de los métodos de investigación al uso (con lo cual se podría hablar, empleando una expresión muy típica de Scholem, de "rebelión en la continuidad"), sobre todo en lo que respecta a la visión de la historia judía transmitida por la Wissenschaft des Judentums ${ }^{3}$. Dicha

\footnotetext{
${ }^{1}$ Cf. G. Scholem, Tagebücher nebst Aufsätzen und Entwürfe, Vol. I: 1913-1917, hrsg. von K. Gründer, H. Kopp-Obersterbrink und F. Niewöhner, Frankfurt a.M., Suhrkamp, 1994; id., Tagebücher nebst Aufsätzen und Entwürfe, Vol. II: 1917-1923, hrsg. von K. Gründer, H. Kopp-Obersterbrink und F. Niewöhner unter Mitwirkung von K.E. Grözinger, Frankfurt a.M., Suhrkamp, 2000.

${ }^{2}$ Véase la famosa carta de Scholem a Salman Shocken, en G. Scholem, Briefe I (1914-1947), hrsg. von I. Shedletzky, München, Beck, 1994, pp. 471-472.

${ }^{3}$ Véase especialmente G. Scholem, "Wissenschaft vom Judentum einst und jetzt", en Bulletin of the Leo Baeck Institute 3, 1960, pp. 10-20; también en id., Judaica I, Frankfurt a.M., Suhrkamp, 1963, pp. 147-164; Cf. además otro texto de G. Scholem en On the Possibility of Jewish Mysticism in our Time \& Other Essays, Philadelphia \& Jerusalem, JPS, 1997, pp. 51-71. La versión original en hebreo de este
} 
visión, como es sabido, debía mucho al lenguaje y al modelo cultural de la emancipación, a su vez reflejo de un milieu que el debate historiográfico sucesivo describió a través de la controvertida expresión "diálogo" o "simbiosis judío-alemana". Una expresión tan fascinante como problemática, que Scholem, si bien con cierta ambigüedad, como testimonian muchos de sus lectores ${ }^{4}$, nunca dejó de contrastar.

Los diarios del joven Scholem revelan hasta qué punto sus raíces culturales se ahondan en ese extraordinario laboratorio de ideas que fue la Alemania de Weimar, donde junto a la recuperación de cierta tradición romántica y prerromántica (por ejemplo Hölderlin, Jean Paul, Novalis, Mörike, por mencionar sólo algunos de los nombres más importantes) ${ }^{5}$ se iba consolidando por vez primera la recepción de la filosofía de la vida de Kierkegaard y Nietzsche. La labor intelectual llevada a cabo por Scholem en su juventud se caracterizaba por el intento de hacer uso de estas y otras lecturas, aplicándolas sin embargo a una nueva manera de interpretar la tarea de la investigación histórica. En este sentido, resulta llamativo que Scholem tomara la decisión de dedicarse a un tema - la Qabbalah - que hasta la fecha había sido considerado pura charlatanería, desprovisto de cualquier interés científico, precisamente porque su alejamiento de la ortodoxia rabínica ponía en peligro la respetabilidad cultural e intelectual tan anhelada por los judíos alemanes. Sin embargo, no se trataba sólo de eso: según Scholem, el hecho de recuperar la herencia del patrimonio místico del judaísmo significaba también abandonar la tradición de la investigación historiográfica propia de las aguas calmas del mundo académico; resultaba necesario adoptar, en cambio, una perspectiva comprometida, capaz de reconocer la justa dignidad a los elementos irracionales de la historia judía y a esas corrientes subterráneas cuya importancia y centralidad Scholem quería resaltar no sólo desde un punto de vista histórico, sino también en el ámbito del debate de su época, respecto al significado de la identidad judía y a la relación entre tradición y modernidad a la luz de una perspectiva post-asimilatoria.

Junto a la historia del misticismo, la gran constelación mesiánica es tal vez el núcleo más relevante en torno al cual, ya desde los primeros años de su investigación, Scholem centró su atención. No cabe ninguna duda, en efecto, de que uno de los resultados más importantes de su indagación ha sido la capacidad de restablecer la centralidad del

ensayo fue publicada en 1944.

${ }^{4}$ Véanse, por ejemplo, G. Mosse, "Gershom Scholem as a German Jew", en Modern Judaism 10, 2 , May 1990, pp. 117-133; S. Aschheim, Scholem, Arendt, Klemperer: Intimate Chronicles in Turbulent Times, Bloomington \& Cincinnati, Indiana University Press, 2001; y P. Mendes-Flohr (ed.), Gershom Scholem: the Man and his Work, Albany, State University of New York Press, 1994.

${ }^{5}$ Cf. M. Löwy, "Messianism in the early work of Gershom Scholem", en New German Critique 83, Special Issue on Walter Benjamin (Spring - Summer 2001), pp. 177-191. 
mesianismo en el ámbito de la historia judía ${ }^{6}$, por supuesto interpretado de forma totalmente distinta respecto a la de Cohen, para quien la idea mesiánica, en una interesante síntesis entre Jerusalén y Königsberg, había cobrado el significado de una continua progresión de la historia humana hacia lo mejor ${ }^{7}$. Scholem, en cambio, intentó mostrar que el mesianismo representa una parte esencial del judaísmo que se concretó de distintas formas, en función de las épocas y del contexto cultural, y cuya presencia en ningún caso puede ser ocultada, a pesar de que durante largas temporadas logró expresarse únicamente a través de corrientes subterráneas. Siguiendo un guión totalmente opuesto respecto al de Cohen, los intereses de Scholem (como también quizás sus simpatías) se dirigieron, ya desde finales de los años veinte, a movimientos místicos de carácter mesiánico y antinómico, como el sabatianismo y el frankismo.

En un contexto así delineado, un análisis de las formas en que se manifiesta el concepto de mesianismo en Scholem resulta sumamente interesante, pues representa tal vez una de las herramientas más eficaces para aclarar, mediante una perspectiva hermenéutica, algunos presupuestos de su investigación, sus intenciones, sus exigencias teóricas y, en cierta medida, también sus preocupaciones filosófico-políticas. Sin duda cabe destacar la dificultad de la tarea, precisamente en virtud de la abundancia de los materiales que sería preciso tomar en consideración.

No hay que olvidarse, por ejemplo, que las primeras referencias a Shabbetai Tzvi se encuentran ya en los diarios, cerca de aquellas páginas de mayo de 1915 en las que Scholem, en plena adolescencia, anotaba su curiosa convicción de ser el Mesías ${ }^{8}$, para retractarse de todo apenas cuatro meses después ${ }^{9}$. En particular, en algunas notas de agosto de 1914, Scholem, que todavía no había cumplido diecisiete años, apunta:

"Cuando Shabbetai Tzvi quiso revelarse a los otros y a sí mismo como el Mesías, se
dirigió hacia la plaza del mercado de Esmirna y, con la cabeza descubierta, pronun-
ció el nombre de Dios. Había una creencia según la cual nadie, excepto el Mesías,
hubiera podido atreverse a nombrar el Tetragrama sin ser fulminado al instante.
Dado que no aconteció nada del género, creyó en sí mismo y la gente creyó en él"10.

\footnotetext{
${ }^{6} \mathrm{Al}$ respecto pueden consultarse las observaciones de J. Dan, "Scholem's view of Jewish Messianism", en Modern Judaism 12, 2, May 1992, pp. 117-128. Cf. también la parte sobre Scholem en P. Bouretz, Testigos del futuro. Filosofía y mesianismo, Madrid, Trotta, 2012, pp. 331-492. En castellano véase también E. Taub, "Historia y neutralización: el mesianismo judío de Gershom Scholem", en Eadem Utraque Europa 9, 14, 2013, pp. 115-136.

${ }^{7}$ Cf. H. Cohen, La Religión de la Razón desde las Fuentes del Judaísmo, trad. de J.A. Ancona Quiroz, Barcelona, Anthropos, 2004.

${ }^{8}$ Cf. G. Scholem, Tagebücher nebst Aufsätzen und Entwürfe, Vol. I: 1913-1917, op. cit., pp. 120-121.

${ }^{9}$ Ibidem, p. 158.

${ }^{10}$ Ibidem, pp. 31-32.
} 
Adicionalmente, en las notas juveniles se hallan referencias aún más interesantes al Mesías y al mesianismo en varios contextos: por ejemplo, en otros muchos pasajes de sus diarios, en particular en las páginas que sirven de comentario al libro de Jonás, donde se confronta el concepto de tiempo mesiánico con la noción de justicia ${ }^{11}$; y finalmente también en ese primer núcleo de consideraciones "a-históricas" (según una definición plasmada por el propio Scholem ${ }^{12}$ ) de su producción representado por las 95 tesis sobre judaísmo y sionismo, que inicialmente fueron pensadas como un regalo para el vigésimo sexto cumpleaños de Walter Benjamin ${ }^{13}$. A la luz de la complejidad de este tema, en el presente artículo nos limitaremos a ilustrar algunas reflexiones introductorias, a fin de evidenciar que el interés de Scholem hacia las distintas declinaciones de lo mesiánico supone mucho más que una simple investigación acerca del fenómeno místico y permite a su vez leer toda su obra en un contexto mucho más amplio, tanto desde una perspectiva histórico-cultural, como en lo concerniente al debate en torno al judaísmo del siglo xx, al cual sin duda pertenecen muchas de las notas scholemianas.

\section{III}

Antes de emigrar a Palestina, en 1923, Scholem ya poseía varios libros dedicados a la figura de Shabbetai Tzvi. Sin embargo, las primeras muestras de un verdadero interés científico por el judaísmo sabatiano se remontan al mes de agosto de 1927, cuando - durante un encuentro en París - Scholem anunció a su amigo Walter Benjamin (en presencia también de Franz Hessel) que durante una estancia de investigación en Oxford había hallado algunos manuscritos sabatianos conservados en la biblioteca bodleiana ${ }^{14}$. En realidad, Scholem ya había revelado su descubrimiento a Benjamin (los dos, por cierto, tuvieron otra ocasión para verse, durante una etapa del viaje a Londres del primero) en una carta perdida cuyo contenido se conoce gracias a la respuesta de Benjamin: Scholem escribió a su amigo para comunicarle que retrasaría su regreso a París porque necesitaba más tiempo para estudiar los manuscritos hallados en Inglaterra. Al año siguiente, Scholem publicó su primer ensayo sobre el sabatianismo en las páginas de un número especial de la revista Der Jude, dedicado al quincuagésimo cumpleaños de su fundador, Martin Buber. Se trataba de un aná-

\footnotetext{
${ }^{11}$ Cf. G. Scholem, Tagebücher nebst Aufsätzen und Entwürfe, Vol. II: 1917-1923, op. cit., pp. 522-532.

${ }^{12}$ Cf. G. Scholem, "Zehn unhistorische Sätze über Kabbalah", en AA.VV., Geist und Werk, Festschrift zum 75. Geburtstag von dr. Daniel Brody, Zürich, Rhein Verlag, 1958, pp. 209-215; también en G. Scholem, Judaica III, Frankfurt a.M., Suhrkamp, 1970, pp. 265-271. Para una traducción al castellano consúltese id., “... todo es cábala”. Diálogo con Jörg Drews, seguido de Diez tesis ahistóricas sobre la Cábala, trad. de M. García-Baró, Madrid, Trotta, 2001.

${ }^{13}$ Cf. G. Scholem, Tagebücher nebst Aufsätzen und Entwürfe, Vol. II: 1917-1923, op. cit., pp. 300-306. ${ }^{14}$ Cf. G. Scholem, Walter Benjamin. Geschichte einer Freundschaft, Frankfurt a.M., Suhrkamp, 1975, p. 171; para una traducción al castellano véase id., Walter Benjamin. Historia de una Amistad, trad. de J. F. Yvars y V. Jarque, Barcelona, Península, 1987.
} 
lisis de la teología del Iggeret Magen 'Avraham de Avraham Cardoso, es decir, el manuscrito hallado y apasionadamente estudiado el año anterior ${ }^{15}$. Después de ese texto y durante su entera existencia, Scholem nunca dejó de enseñar y escribir sobre el sabatianismo, volcando la mayor parte de sus esfuerzos hacia el intento de arrojar luz sobre los distintos aspectos de lo que tal vez fue el episodio más desbaratador de la historia del judaísmo de la diáspora. En efecto, la importancia y la unicidad de la contribución de Scholem a este ámbito de estudios se concretó en su monumental biografía sobre Shabbetai Tzvi - publicada en hebreo en 1957 y después en inglés, en la traducción de Raphael Zwi Werblowsky, en 1973 -, uno de los estudios judíos más significativos del siglo Xx, como ha hecho notar Moshe Idel ${ }^{16}$.

Es importante subrayar que Scholem siempre consideró el sabatianismo una expresión de la energía vital del judaísmo y no un cuerpo extraño a dicha tradición, como trató de hacer, en cambio, la vulgata rabínica. En contraposición a ese intento de damnatio memoriae, Scholem estaba convencido de que el sabatianismo había sido la verdadera fuerza motriz del proceso de secularización judía, es decir, el impulso que había favorecido la aceptación, por parte de los judíos, de la liberación de las ataduras del gueto. Sin embargo, no es fácil establecer si - y en qué medida - Scholem, en su análisis del movimiento sabatiano, quedó impresionado y seducido por el espíritu anárquico de su fundador, hasta casi asumirlo como su propio alter ego, o si más bien fue Scholem quien trasladó a la figura de Shabbetai Tzvi sus propias simpatías juveniles por el anarquismo; se trata, sin lugar a dudas, de una cuestión muy intrincada, que reaparece constantemente en buena parte de la literatura post-scholemiana. En cualquier caso, resulta evidente que la presentación del mesianismo sabatiano que hizo Scholem, especialmente en uno de sus textos más significativos - el artículo de 1936 titulado emblemáticamente "Redención a través del pecado" 17 -, insistía en sus aspectos anarquistas y de ruptura con la tradición, dando cuenta al mismo tiempo de su incidencia en el desarrollo de la historia judía.

A pesar de la perplejidad que el propio Scholem constató a este respecto, dicha lectura parece confirmar la interpretación de la historiografía scholemiana bajo el signo de la "anarquía" o de la "contra-historia", según comentó David Biale, autor de la primera

\footnotetext{
${ }^{15}$ Cf. G. Scholem, Die Theologie des Sabbatianismus im Lichte Abraham Cardosos, en id., Judaica I, op. cit., pp. 119-146.

${ }_{16}$ M. Idel, Old Worlds, New Mirrors. On Jewish Mysticism and Twentieth-Century Thought, Philadelphia, University of Pennsylvania Press, 2010, p. 9.

${ }^{17}$ El título original en hebreo es "Mitzwah ha-ba'ah ba-'averah". Scholem hace ya referencia a este ensayo en una carta a Walter Benjamin del 18 de diciembre de 1935. Véase al respecto W. Benjamin - G. Scholem, Briefwechsel, Frankfurt a.M., Suhrkamp, 1980, pp. 212-213 (en castellano: Correspondencia 1933-1940, trad. de R. Lupiani, Madrid, Trotta, 2011). Según la correspondencia, Benjamin, que no sabía hebreo, pudo leer una versión resumida en alemán de este trabajo, que fue publicado en el almanaque de la Schocken Verlag del 5697 (1936/37).
} 
biografia intelectual de Scholem ${ }^{18}$. Sin embargo, esta tensión anarquista es muy difícil de comprender. Ciertamente, Scholem consideraba su propio anarquismo de forma contrapuesta al de Buber, a quien de hecho acusaba de no tener en cuenta la dimensión dialéctica. Semejante anarquismo, en sus consecuencias religiosas - tal como lo entendía Scholem - bajo ningún concepto involucraba una capitulación frente a la ortodoxia ni tampoco una suerte de complejo de inferioridad respecto a ella:

\begin{abstract}
"Somos todos anarquistas, incluso si tal anarquía es sólo una fase de transición y nosotros somos el ejemplo viviente de que dicha posición no nos excluye del judaísmo. No somos una generación sin preceptos, aunque nos falte autoridad (samkhut). No experimento una sensación de inferioridad respecto a los ortodoxos. Nosotros no tenemos menos legitimidad que nuestros padres, sino que ellos gozaban de una definición más clara. Quizá seamos anarquistas, pero estamos en contra de la anarquía"19.
\end{abstract}

Ahora bien, considerada dicha actitud anarquista y, por decirlo así, cierta predilección por movimientos como el sabatianismo, ¿cuál es la postura de Scholem frente al mesianismo en general? La interpretación más en boga parece otorgar un papel dominante al paradigma de la crisis. En particular, la propuesta scholemiana se caracteriza por ofrecer una declinación "apocalíptica" del mesianismo (incluso en abierta contraposición con las visiones precedentes), que tiende a enfatizar el carácter catastrófico del proceso de redención:

"Por su origen y esencia, el mesianismo judío es una teoría de la catástrofe, cosa que nunca se subrayará demasiado. Esta teoría hace hincapié en el elemento revolucionario y demoledor que se encierra en el tránsito del presente histórico al futuro mesiánico"²0.

\footnotetext{
${ }^{18}$ Cf. Biale, Gershom Scholem. Kabbalah and Counter-history, Cambridge \& London, Harvard University Press, $1979^{1}, 1982^{2}$. Se puede deducir que Scholem no estaba completamente convencido de esta interpretación gracias a un párrafo de una carta ( $\sin$ fecha, de la que tan sólo queda la ultima página, la veintisiete) conservada en el archivo Scholem de la Biblioteca Nacional de Israel. La carta estaba dirigida a Biale, quien a la sazón trabajaba en su tesis de doctorado sobre el propio Scholem. En particular y comentando algunos puntos del manuscrito de Biale, Scholem intentaba distinguir claramente entre su propio trabajo científico sobre la Qabbalah y sus escritos ahistóricos: "You say that I defined the Kabbala 'as an anarchistic tradition', which is not true. It is you who does this: I never did. [...] You are consistently confusing my unhistoric reflections with my historic research and its results. If you only would say so!!' (Biblioteca Nacional de Israel, Arch. 4, 1599/277-I/127, Jerusalén).

${ }^{19}$ G. Scholem, 'Od Davar, Tel Aviv, 'Am 'Oved, 1989, pp. 95-97. Cf. también P. Mendes-Flohr, Divided Passions. Jewish Intellectuals and the Experience of Modernity, Detroit, Wayne State University Press, 1991, p. 400. Se trata de una parte del protocolo resultante de la reunión del grupo "Ha-'ol" [el yugo], del cual Scholem era miembro, que tuvo lugar el día 13 de julio de 1939.

${ }^{20}$ G. Scholem Para comprender la idea mesiánica en el judaísmo, en G. Scholem, Conceptos básicos del judaísmo, trad. de J.L. Barbero, Madrid, Trotta, 2008³, pp. 99-135, aquí p. 106.
} 
Según Scholem, ni en la Biblia ni en los autores apocalípticos se encuentra una progresión histórica capaz de conducir a la redención; esta peculiar declinación del mesianismo es la misma que Scholem reconocía también en la contemporaneidad. La redención, en cambio, debería ser considerada como un evento repentino, una ruptura del continuum de la historia, según una concepción que - tal como Stéphane Mosès notó magistralmente ${ }^{21}$ - consigue mantener bajo el mismo signo los recorridos, en cierto sentido muy diferentes, de Scholem, Benjamin y Rosenzweig. Fue el propio Scholem quien ofreció una descripción del evento mesiánico que podríamos considerar, con razón, como la más "iluminadora":

"La salvación es ante todo una irrupción de la trascendencia en la historia, una irrupción en la que la historia misma es aniquilada, aunque en su hundimiento se transforme al ser iluminada por una luz que viene de otra parte"22.

A pesar de que conocía otras formas de mesianismo (o que, según una acepción más amplia respecto a la suya, podrían ser consideradas como tales), como ha notado Idel $^{23}$, Scholem prefirió ocuparse exclusivamente de una de las muchas expresiones particulares en las que el mesianismo judío cristalizó, precisamente porque su obsesión por el imaginario de la catástrofe ${ }^{24}$ le indujo a considerar "menos auténticas" algunas manifestaciones circunscritas al plano del individuo y que, por lo tanto, no tuvieron repercusiones históricas.

"Hay un punto importante en el que el apocalipsis secularizado o teoría catastrófica de la revolución (que tan importante papel desempeña en los debates actuales) sigue vinculado a su punto de partida en la teología judía, del que procede, aunque no lo confiese. Es éste el rechazo a la interiorización radical de la redención. No es que en la historia del judaísmo hayan faltado intentos de descubrir también una dimensión semejante en el mesianismo judío (especialmente, y como era de esperar, en la mística). Pero, en todas sus configuraciones históricas, el judaísmo ha rechazado completamente la tesis de una interiorización químicamente pura de la redención.

\footnotetext{
${ }^{21}$ Cf. S. Mosès, El ángel de la historia. Rosenzweig, Benjamin, Scholem, trad. de A. Martorell, Madrid, Cátedra, 1997.

${ }^{22}$ G. Scholem, Para comprender la idea mesiánica en el judaísmo, op. cit., pp. 108-109. Cabe destacar que, en el segundo ensayo de la colección The Messianic Idea in Judaism, cuyo título es "La idea mesiánica en la Qabbalah", Scholem encuentre curiosamente en el misticismo las raíces de la concepción del ideal mesiánico como infinito progreso e infinita perfectibilidad del género humano.

${ }^{23}$ Para describir la visión scholemiana del mesianismo Moshe Idel ha propuesto utilizar la expresión "diacronismo monocromático": M. Idel, Messianic Mystics, New Haven, Yale University Press, 1998, p. 33.

${ }^{24} \mathrm{Al}$ respecto véase H. Bloom (ed.), Gershom Scholem, New York-New Haven-Philadelphia, Chelsea House Publishers, 1987, p. 207-220.
} 
Una interioridad que no se expresara también en lo exterior, que no se vinculara a ello de principio a fin, no valía aquí absolutamente nada. El avance hacia el núcleo es aquí, a la vez, avance hacia fuera" ${ }^{25}$.

Es posible que este paradigma de la crisis, tan predominante también en la interpretación scholemiana de la Qabbalah y del judaísmo en sí, descienda de un sentimiento del ocaso de Occidente y de aquella experiencia de desencantamiento del mundo que caracteriza la modernidad y, en particular, la modernidad judía; en este sentido, pues, gozaría de crédito la hipótesis según la cual la perspectiva de Scholem puede entrar en contacto de manera muy pertinente con otros recorridos filosóficos parecidos, que tienen su origen en el mundo judío-alemán de la época de Weimar ${ }^{26}$.

\section{IV}

El escrito más conocido que Scholem ha dedicado al tema del mesianismo es probablemente Para comprender la idea mesiánica en el judaísmo, fruto de una conferencia pronunciada en 1959 en los coloquios de Eranos ${ }^{27}$. Se trata de un ensayo importante que, según hace notar Joseph Dan, constituye el único texto sobre el mesianismo en el que Scholem desarrolla una investigación sobre la idea mesiánica desde la época bíblica hasta al siglo doce sin casi mencionar el misticismo ${ }^{28}$. Dada su relativa concisión, el texto permite que se identifiquen algunos de los puntos clave de la reflexión scholemiana $y$, en particular, la dimensión polar de un mesianismo que contiene elementos de utopía y de conservación en una dialéctica entre negación y constante referencia a la tradición.

Este ensayo también ha suscitado algunas de las críticas más fuertes a la literatura scholemiana. Es el caso, por ejemplo, de las observaciones de Jacob Taubes, quien, como es sabido, tendrá con Scholem una relación tormentosa en el ámbito personal aún antes que en el académico ${ }^{29}$. Hijo del Gran Rabino de Zúrich y rabino él mismo,

\footnotetext{
${ }^{25}$ G. Scholem, Hay un misterio en el mundo. Tradición y secularización. Una conferencia y un diálogo, trad. de M. Abella, Madrid, Trotta, 2006, pp. 40-41.

${ }^{26}$ Podrían evocarse aquí, por ejemplo, los nombres de Leo Strauss y Ernst Bloch.

${ }^{27}$ Con respecto a la participación de Scholem en los encuentros de Eranos consúltese N. Zadoff, MiBerlin leYerushalayym uweChazarah. Gershom Scholem ben Israel weGermania [De Berlín a Jerusalén y regreso. Gershom Scholem entre Israel y Alemania], Jerusalén, Carmel, 2015, pp. 294-331. ${ }^{28}$ Cf. Dan, "Gershom Scholem and Jewish Messianism", en P. Mendes-Flohr (ed.), Gershom Scholem: the Man and his Work, op. cit., p. 74.

${ }^{29}$ Sobre la figura de Taubes cf. M. Treml, "Reinventing the Canonical: The Radical Thinking of Jacob Taubes", en E. Goebel y S. Weigel (eds.), "Escape to Life". German Intellectuals in New York: A Compendium on Exile after 1933, Berlin, De Gruyter, 2012, pp. 457-478; en castellano véase G. Hervás, "Secularización y mesianismo. El pensamiento político de Jacob Taubes", en Diánoia 52, 68, 2012, pp. 81-111.
} 
tras haber publicado en 1947 su tesis Escatología Occidental ${ }^{30}$ (única monografía que el autor publicó en vida), Taubes fue contratado en el Jewish Theological Seminary de Nueva York. Después de un paréntesis muy breve de colaboración con Scholem en Jerusalén, que terminó de la peor de las maneras, volvió a Estados Unidos, primero a Harvard y luego a Columbia, donde impartió clases de Historia y Filosofía de las Religiones. Durante los años sesenta, Taubes fue invitado a dirigir el Departamento de Hermenéutica en la Freie Universität, que bajo su mando se convirtió en el centro del movimiento estudiantil berlinés. Su voluntad de participar activamente en la lucha política, sus guiños al anarquismo y su conocimiento vivo (no sólo derivado del estudio académico) de la tradición rabínica constituyen los elementos imprescindibles para comprender tanto el pensamiento de Taubes - sin tener en cuenta su idiosincrasia y el aura mítica que lo rodeó en vida - como su capacidad de poner en evidencia algunas cuestiones no resueltas del pensamiento de Scholem. Entre 1977 y 1982 Taubes publicó tres artículos ${ }^{31}$ decididamente críticos con respecto a la descripción scholemiana del mesianismo que lo llevaría, en consecuencia, a asumir una posición fuertemente polémica en referencia a la cuestión de la herencia del pensamiento benjaminiano, opuesta al frente Scholem-Adorno. Aun escasamente argumentadas en algunos puntos y en cierta medida demasiado simplistas, las críticas de Taubes tienen el mérito de volver a suscitar un debate sobre el pensamiento scholemiano en clave filosófica y sobre todo teológico-política.

El punto de partida metodológico del análisis de Taubes es que no son necesarias más investigaciones sobre el material presentado por Scholem para poder ofrecer una lectura distinta e incluso opuesta de los acontecimientos descritos por él. De hecho, Scholem llegó a una cierta interpretación por el uso "demasiado rígido" de su aparato categorial y sobre todo, como se verá, en razón de de sus convicciones políticas sobre el futuro del judaísmo. En primer lugar, se puso en evidencia la contraposición delineada por Scholem entre judaísmo y cristianismo frente a la experiencia del mesianismo, contraposición enteramente establecida, una vez más, sobre la relación interioridad/ exterioridad:

"En todas sus modalidades y formas el judaísmo siempre ha concebido y defendido la salvación como un proceso, en resumen, que se decide en el mundo de lo visible

\footnotetext{
${ }^{30}$ J. Taubes, Escatología occidental, trad. de C. Pivetta, Buenos Aires, Miño y Davila, 2010.

${ }^{31}$ J. Taubes, "Der liebe Gott steckt im Detail. Gershom Scholem und die messianische Verheißung", en Die Welt 288, 10.12.1977, p. 1; id., "Scholem's Theses on Messianism Reconsidered", en Social Science Information 21, 4-5, 1982, pp. 665-675; id., "El mesianismo y su precio", en id., Del culto a la cultura. Elementos para una crítica de la razón histórica, trad. de S. Villegas, Buenos Aires, Katz, 2007, pp. 45-51. Una versión ligeramente diferente de este ultimo ensayo, junto con la correspondencia entre Scholem y Taubes, se encuentran en id., Der Preis des Messianismus: Briefe von Jacob Taubes an Gershom Scholem und andere Materialien, hrsg. von E. Stimilli, Würzburg, Königshausen \& Neumann, 2006.
} 
y que no puede pensarse sin esa proyección visible. Por el contrario, el cristianismo concibe la salvación como un proceso del ámbito 'espiritual' e invisible, que se desarrolla en el alma, en el mundo individual de cada persona, y que provoca una conversión interna, sin correspondencia necesaria en el mundo externo" 32 .

Se trata, como puede apreciarse, de una lectura que constituye sólo uno de los intentos de trazar una diferencia entre judaísmo y cristianismo que se pueden encontrar en numerosos textos del pensamiento judío del siglo xx (por ejemplo en Buber, Klausner o Baeck). Sin embargo, Taubes se sirve de esta distinción scholemiana, que en el ensayo en cuestión ocupa en realidad poco espacio, y evidencia sus limitaciones, adelantando ya mucho de lo que, posteriormente, se podrá leer en sus lecciones sobre la Epístola a los Romanos, aparecidas póstumamente ${ }^{33}$. Taubes afirma con convicción que la interiorización no constituye de ninguna forma una línea de demarcación entre judaísmo y cristianismo; más bien indicaría una crisis totalmente interna a la escatología judía en el momento de su actualización. Como se pregunta el propio Taubes: “¿De qué otra manera puede definirse la redención, después de que el mesías no ha redimido el mundo exterior, sino trasladándola a la interioridad?" 34 . En defensa de Scholem, sin embargo, no se puede negar que una mirada más atenta a la labor scholemiana, y en particular a los otros ensayos que conforman el volumen The Messianic Idea in Judaism, restaría rigor a la crítica taubesiana o cuando menos pondría de relieve el hecho de que Scholem era perfectamente consciente del tipo de problemática planteada por Taubes ${ }^{35}$.

La parte más relevante del ensayo scholemiano, objeto de las críticas de Taubes, sin embargo, parecen ser las páginas en las que Scholem introduce el tema del "precio del mesianismo", considerado el fruto de un inaceptable "cálculo psicoeconómico" incapaz de superar un examen histórico. Además de en el final del ensayo de 1959 (luego retomado también en la conclusión de una ponencia pronunciada en la clausura de un congreso sobre la idea mesiánica en el judaísmo y dedicada a él en 1977, en ocasión de sus 80 años) y en numerosas entrevistas, la expresión será utilizada por Scholem también en el prefacio de la edición inglesa de la biografía de Shabbetai Tzvi ${ }^{36}$. El precio del mesianismo, según Moshe Idel, uno de los más "atractivos ejemplos de la axiología scholemiana"37, correspondería a lo que pagó el pueblo judío por haber seguido siendo fiel en el tiempo a la idea mesiánica:

\footnotetext{
${ }^{32}$ G. Scholem, Para comprender la idea mesiánica en el judaísmo, op. cit., p. 99.

${ }^{33} \mathrm{Cf}$. J. Taubes, La teología política de Pablo, trad. de M. García-Baró, Madrid, Trotta, 2007.

${ }^{34} \mathrm{~J}$. Taubes, El mesianismo y su precio, op. cit., p. 46.

${ }^{35}$ Consúltense, por ejemplo, las observaciones de Scholem en "La crisis de la tradición en el mesianismo judío”, en The Messianic Idea in Judaism, op. cit., pp. 49-77, especialmente p. 52; 58-59.

${ }^{36}$ G. Scholem, Sabbetai Sevi. The Mystical Messiah, traducido por Z. Werblowsky, Princeton, Princeton University Press, 1973, p. xii-xiii.

${ }^{37}$ M. Idel, Absorbing Perfections. Kabbalah and Interpretation, New Haven, Yale University Press, 2002, p. 506 n. 84.
} 
"La grandeza de la idea mesiánica se corresponde con la infinita debilidad de la historia judía, que desde el Exilio no ha estado preparada para comprometerse en la arena de lo histórico. Su historia tiene la debilidad de lo efímero, lo provisorio, pero que en cambio nunca se agota. Porque la idea mesiánica no es únicamente consuelo y esperanza. Cada intento de su consumación abre abismos que llevan al absurdo cualquiera de sus formas [...] Así la idea mesiánica ha forzado al judaísmo a 'vivir a plazos', es decir, sin que nada de lo que se haga pueda ser definitivo o completo" 38 .

La perspectiva de Taubes, no obstante, es radicalmente opuesta: si el judaísmo de la diáspora ha vivido fuera de la historia, esto no se debe al mesianismo, sino más bien a la hegemonía rabínica. Contrariamente a lo que pensaba Scholem, cualquier esfuerzo para revitalizar la idea mesiánica corresponde históricamente, según afirma Taubes, a un intento de hacer un salto en la historia. La cuestión teológico-política, concerniente al Estado de Israel e implícita en estas notas taubesianas, es aquí fácilmente reconocible y, de hecho, se explicita mediante una crítica directa a Scholem, tan feroz que se acusa al profesor de historia de aportar una falsa representación de los hechos:

"Lo que induce Scholem a negar ciertas correlaciones parece ser su deseo de liberar el experimento utópico sionista de todas las implicaciones apocalípticas y mesiánicas. Preocupado por las consecuencias destructivas de la fantasía mesiánica, Scholem llegó a negar la relevancia de los elementos mesiánicos en el utópico regreso sionista, falsificando así la historia" ${ }^{39}$.

En este sentido, lo que Taubes reprocha a la reflexión scholemiana, en última instancia, es que quería absolver al sionismo, liberándolo de cualquier implicación en dinámicas mesiánicas. Taubes será aún más cáustico, si cabe, en la conclusión de sus artículos, al escribir: "El historiador no puede hacer más que llevar correctamente los libros. Pero en la actual situación espiritual y política del pueblo judío, puede, con ello, plantear un problema y señalar un peligro"40. No sería incorrecto, a mi modo de ver, identificar en esta insinuación un ulterior intento envenenado de distanciarse de las reflexiones sobre la escasa influencia política de los historiadores expresadas por Scholem en dos entrevistas de $1980^{41}$, uno y dos años antes, por tanto, de que Taubes escribiera estos artículos.

\footnotetext{
${ }^{38}$ G. Scholem, Para comprender la idea mesiánica en el judaísmo, op. cit., p. 134.

${ }^{39}$ J. Taubes, Der Preis des Messianismus: Briefe von Jacob Taubes an Gershom Scholem, op. cit., p. 38.

${ }^{40} \mathrm{~J}$. Taubes, El mesianismo y su precio, op. cit., p. 51.

${ }^{41}$ Se trata de dos entrevistas a Scholem, en las que se posicionaba negativamente respecto de la carta abierta, publicada en "Ha'aretz", del historiador Jacob Talmon a Menachem Begin, en la cual las enseñanzas del pasado se usaban como medio para criticar la política del coetáneo primer ministro israelí. Respondiendo a Biale, que le preguntaba si estaba de acuerdo con Talmon respecto a la posibilidad de que los profesores de historia pudieran enseñar algo a los políticos, Scholem afirmó: "I am very skeptical about that, although I know that Jacob Talmon thinks otherwise. [...] In any event, history never repeats itself in exactly the same way. I doubt whether professors of history can teach
} 
También la relación entre sionismo y mesianismo en Scholem, retomada por Taubes, constituye ciertamente uno de los problemas que deben todavía analizarse de manera sistemática, si bien su discusión aquí no tiene suficiente cabida. Sin embargo, sí merece la pena señalar que, a pesar de algunas ambigüedades y de cierta desilu$\operatorname{sión}^{42}$, ya desde el final de los años veinte, en lo que atañe a la propia opción sionista (moldeada sobre el modelo del sionismo cultural de Ahad Ha'am), Scholem estuvo siempre atento a evidenciar que, más allá de una aplicación impropia (y peligrosa) de una determinada fraseología mesiánica ${ }^{43}$, el sionismo no tenía nada que ver con el mesianismo, tratándose el primero de un movimiento obligado a rendir cuentas a la realidad histórica, mientras que el segundo, en cambio, quedaba confinado a la esfera metafísica ${ }^{44}$.

such things to anyone. I have been a professor of history too long to believe that": Biale, "The Threat of Messianism: An Interview with Gershom Scholem", en The New York Review of Books, 14 August 1980. También con ocasión de otra entrevista Scholem reafirmará su posición, declarándose "menos optimista que Talmon con respecto a que los profesores puedan influir en los acontecimientos": cf. Howe, I., "Interview with Gershom Scholem: The Only Thing in My Life I have Never Doubted is The Existence of God", en Present Tense 8, 1, Autumn 1980, pp. 53-57.

${ }^{42}$ Cf. F. Niewöhner, "Im Brennpunkt der Historie. Selbstkritik des Zionismus: Gershom Scholems esoterische Aufzeichnungen der Jahre 1930/31", en Frankfurter Allgemeine Zeitung, 29.10.1997, 251, p. N6. Véase también E. Lucca, "Decadenza del linguaggio e crisi del sionismo nel giovane Scholem", en Rivista Italiana di Filosofia del Linguaggio 8, 2, 2014, pp. 171-181.

${ }^{43}$ No es casualidad que Scholem ya se hubiera referido a "los eslóganes mesiánicos del sionismo" en la conclusión de su primer trabajo dedicado al sabatianismo, Die Theologie des Sabbatianismus im Lichte Abraham Cardosos, op. cit. Véase al respecto también la entrevista con D. Biale del 1980: "The Jews have always had a fatal attraction to messianism. The Jewish involvement in communism, for instance, was definitely a consequence of Jewish messianism. Zionism is no exception. Today we have the Gush Emunim which is definitely a messianic group. They use biblical verses for political purposes. Whenever messianism is introduced into politics, it becomes a very dangerous business. It can only lead to disaster": Biale, "The Threat of Messianism: An Interview with Gershom Scholem", op. cit. En la misma entrevista Scholem no duda en comparar los miembros de Gush Emunim con los sabatianos. ${ }^{44}$ Véase, por ejemplo, G. Scholem, On the Possibility of Jewish Mysticism in our Time \& other Essays, op. cit., p. 116: "[...] I consider it the pride of Zionism that it is not a messianic movement. It is a great error, therefore - for which Zionism may have to pay dearly - if the movement attributes to itself messianic significance. Messianic movements are apt to fail. Zionism is rather a movement within the mundane, immanent process of history; Zionism does not seek the end of history, but takes responsibility within the history of an unredeemed and unmessianic world". Por otra parte téngase en cuenta la posición contraria de Taubes: "El reclamo mesiánico 'fue convocado básicamente' a partir del horror y la destrucción de los judíos de Europa e hizo posible que la realidad política del Estado de Israel se cargara de locas fantasías apocalípticas": J. Taubes, El mesianismo y su precio, op. cit., p. 51. 